\title{
Commentary
}

\section{Patient M: Considerations for use of atypical antipsychotics in borderline personality disorder}

\section{TAMARA L. GAY, Department of Psychiatry, University of Michigan, Ann Arbor, MI, USA}

Dr Casare Maffei has provided us with a fascinating case report (patient M). Dr Maffei describes this complex and challenging patient and presents an excellent synopsis of her developmental, clinical, family and social history. Her varied and evolving symptomatology includes disordered binge eating, great interpersonal difficulties, severe self-mutilating behaviours including suicide attempts, and extreme substance abuse of prescription medications. Also present are depersonalization experiences and somatic delusional beliefs ( $M$ considers food 'dirty' and believes that in some way food will damage her bowels). Adding to the treatment challenge is that the patient often gives vague and/or simplistic descriptions to her therapists and doctors, apparently being unable to utilize higher-order abstract reasoning and communication skills in therapeutic venues.

We are introduced to $\mathrm{M}$ as a 31-year-old unmarried female, living in her parents' home. She has successfully completed a law degree, but has never worked in this field. She took 9 years to complete her degree. Great interpersonal difficulties interfere with her current job as a secretary. She sought treatment at age 24 because of severe selfmutilating behaviours, mainly forearm cutting and burning, and impulsive behaviours in multiple areas, namely binge eating and medication abuse.

$\mathrm{M}$ has experienced a wide variety of psychiatric symptoms throughout her life. These include, in order of presentation, the following: obsessions and compulsions; beginning at age 11 , self-injurious behaviours; and beginning at age 16, disordered eating behaviour, with binging, laxative abuse and induced vomiting. She has had numerous suicidal or parasuicidal attempts/gestures by drug overdose. Two of these attempts required hospitalization. The patient has also experienced dissociative type experiences, and she describes magical thinking about being able to be in contact with the dead.

Patient M's Axis II diagnosis is borderline personality disorder (BPD) with schizotypal traits. She meets DSM-IV criteria, via the Structured Clinical Interview D (SCID-II). She fulfils seven of the nine BPD criteria, in addition to four schizotypal personality disorder criteria. The use of antipsychotic medications in schizotypal personality disorder has been studied for the past 25 years, and for at least the past 20 years, the use of these medications in BPD has also been studied. Soloff's 1998 review suggested that low doses of first-generation antipsychotic drugs were effective in reducing cognitive perceptual abnormalities in both borderline and schizotypal personality disorders. M's psychotic and parapsychotic symptoms, specifically somatic delusions, odd beliefs, magical thinking and unusual perceptual experiences argue for trials of antipsychotic medication. Early in her treatment course, she received first-generation antipsychotic agents, including haloperidol and 
chlorpromazine. She was noted to 'misuse' chlorpromazine by taking much higher doses than prescribed and resorted to stealing and forging prescriptions. M's chaotic and worrisome symptoms continued, and within a year of initiation of haloperidol and chlorpromazine, as well as prescription drugs from other classes, she stopped all psychiatric treatment.

A very condensed account of her treatment from ages 24-31 includes short hospital stays, brief trials of outpatient psychotherapy, pharmacotherapy and day hospitalization. M's usual pattern was to terminate all treatments early and to avoid restarting treatment. She also self-imposed a 4-year treatment hiatus from ages 25-29. While pharmacotherapy was started coincident with her first psychiatric contact at age 24 , she did not receive atypical antipsychotic medications until very late in the described treatment course. Exploration of her treatment experience with several atypical antipsychotic medications will follow, in addition to the research findings that have provided a reasonable rationale for choosing drugs in this class to treat BPD.

Clozaril was the first atypical (novel) antipsychotic administered to our patient, for several cited reasons. One was the thought that the necessity of frequent blood draws to monitor white blood cell counts would make attendance at regular medical visits imperative. Also, it was hoped that previous misuse of prescribed medication, which had been so problematic for this patient, would be minimized by her agreement to this plan. M responded well to this first chosen atypical antipsychotic medication; she stopped self-cutting behaviours and reduced laxative abuse. Evidence for use of clozapine in BPD patients with psychotic features and self-mutilation is clear (Chengappa, Ebeling, Kang, Levine, \& Parepally, 1999). A preliminary study by Frankenburg and Zanarini (1993) examined the effects of clozapine on 15 patients with 'prolonged and/or pronounced psychotic symptoms of an atypical nature'. On the Brief Psychiatric Rating Scale (BPRS), subjects' ratings decreased significantly after 2-9 months of treatment for positive, negative and overall symptomatology. Another early open study of low-dose clozapine was published by Benedetti, Sforzini, Colombo, Maffei, and Smeraldi (1998). Twelve borderline personality disordered inpatients with psychotic-like symptoms were studied. The clozapine dose was up titrated in each individual patient using complete disappearance of psychotic symptoms as the dosage endpoint. BPRSs were performed by non-blinded clinicians and showed statistically significant decreases in BPRS scores within the first 3 weeks of treatment, indicating decreases in psychotic-like symptoms. Impulsive behaviours and depression-related symptoms also diminished. In this publication, Benedetti et al. discuss the rapid decrease in psychotic symptoms in addition to broad improvement across all symptom clusters, achieving these results with very low doses of clozapine (25-100 mg). A recently published meta-analyses of multiple drug classes for treatment of severe personality disorders reveals that no randomized controlled trial (RCT) studying clozapine for treatment of personality disorder has been done to date (Ingenhoven, Lafay, Rinne, Passchier, \& Duivenvoorden, 2010). Perhaps the most obvious reason for the lack of RCTs for clozapine in the treatment of BPD is the aforementioned potentially life-threatening side effect of agranulocytosis (Lambertenghi Deliliers, 2000). In clinical practice, clozapine is reserved as a third- or fourth-line agent to treat psychosis as it is much more cumbersome to use in comparison with other drugs in its class. It would be logical to assume that researchers have taken these difficulties of clinical use into consideration and have decided not to pursue systematic RCTs of clozapine.

The second atypical antipsychotic given to our patient was risperidone. There have not been RCTs of risperidone in patients with BPD, but there is one RCT of this agent, published by Koenigsberg et al. (2003), for the treatment of schizotypal personality disorder. This 9-week study included 23 outpatients with only a very low percentage $(20 \%)$ having co-morbid BPD. The starting dose of risperidone in this study was $0.25 \mathrm{mg}$, 
with the highest dose being $2.0 \mathrm{mg}$. This study utilized the Positive and Negative Syndrome Scale (PANSS), and showed that subjects receiving active medication had significantly lower scores on the PANSS negative, positive and general subscales by the end of the study. As patient $M$ had significant schizotypal features, this drug was a very logical second choice based on the available literature. However, the patient's symptoms appeared to worsen on low-dose risperidone ( $0.5 \mathrm{mg} /$ day), with two incidents of self-cutting; therefore, after 3 weeks of risperidone treatment, she was switched to olanzapine. Risperidone has the highest incidence among the atypicals of extrapyramidal symptoms (EPS) (Weiden, 2007). Rapidly escalating dosage also puts patients at greater risk for this side effect. Also, specific patient qualities, such as male gender and young age, put patients at higher risk for EPS. On the other hand, when taking metabolic effects into account, risperidone has a relatively low risk of causing diabetes and obesity (Murashita et al., 2007).

Olanzapine is the best systematically studied atypical antipsychotic medication via RCTs (Abraham \& Calabrese, 2008). It was the third atypical antipsychotic medication chosen by M's psychiatrist after withdrawal of clozaril was necessitated by the appearance of the serious side effect of agranulocytosis, and a brief trial of low-dose risperidone was regarded as a treatment failure. RCTs using olanzapine to treat personality-disordered patients began to appear in the literature in 2001 (Zanarini \& Frankenburg, 2001). While this first study's sample contained only 28 patients diagnosed with BPD, the outcome measures were very enlightening. The authors found that on self-ratings of paranoia, anxiety, anger/hostility and interpersonal sensitivity using scales on the Symptom Checklist-90 (SCL-90), there was a statistically significant improvement over time (6 months) for the olanzapine-treated group. However, there was no difference in self-ratings on the depression scale of the SCL-90 between the olanzapine and control groups. Bogenschutz and Nurnberg (2004) published a 12-week placebo-controlled trial with olanzapine, using adjusted dosing with a range of 2.5-20 mg/day. The authors used outcome measures of the Clinical Global Impression Scale (CGI) applied to each of the DSM-IV criteria for BPD (CGI-BPD) as well as an overall CGI rating. By week 4, there was significant separation on these measures between the olanzapine and control groups. A third and very interesting study utilizing olanzapine was done by Soler et al. (2005). Their methodology involved initial treatment of 60 patients with dialectical behaviour therapy (DBT), and while continuing this structured psychotherapeutic treatment, the subjects were randomized into DBT alone and DBT plus olanzapine groups. Findings of this 12-week outpatient trial included greater improvement in the olanzapine plus DBT cohort on both the Hamilton Depression Rating Scale (HAM-D), and the Hamilton Anxiety Rating Scale (HAM-A). There was also a greater reduction in reports of aggressive/impulsive behaviour in the olanzapine-treated group. In each of the above studies, statistically significant greater weight gain in olanzapine groups was also a reported outcome. In our study of patient $\mathrm{M}$, we learn that the initiation of olanzapine had 'satisfying results'. Specifically, Dr Maffei explains that this patient has shown slow but progressive improvement, with better control of impulsivity and her behaviours of substance and laxative abuse ended during the most recent 2 months of treatment described by this case report. Soler et al. (2005) reported significant weight gain with olanzapine, without a dosedependent relationship. M's metabolic status should be monitored closely as long as she remains on any atypical antipsychotic drug. Patients with disordered eating show decreased adherence to a medication when weight gain is a possible side effect.

The atypical antipsychotic aripiprazole was not utilized in M's treatment. However, for completeness, a description of the RCT done by Nickel, Loew, and Gil (2007) is included here. The authors recognized that given the novel mechanism of action of aripiprazole and the evidence for its efficacy in the treatments of schizophrenia, schizoaf- 
fective disorder, manic and mixed states of bipolar disorder, it was reasonable to study this medication's usefulness in treating BPD. A double-blind, placebo-controlled study involving 52 subjects with confirmed BPD diagnosis by SCID-II was completed over an 8-week period. Improvement was measured by the SCL-90-R, HAM-D, HAM-A and the State-Trait Anger Expression Inventory. Significant separation between the active treatment and placebo groups was found using each of the above measurements. The authors found that all SCL-90-R scores were improved in the aripiprazole group, but were particularly improved on the obsessive-compulsive, insecurity in social contacts, depression, anxiety, aggressiveness/hostility, phobic anxiety, paranoid thinking and psychoticism scales.

Our patient's presentation can be characterized as variable, complex and severe. Most of her symptoms including her compulsive neatness, social avoidance, suicide attempts, paranormal beliefs/ magical thinking, paranoia about food and contamination, and her early aggressive behaviour with her brother as well as self-aggression would each fit into one or several of the above domains. Therefore, it is reasonable to think of aripiprazole as a possible future treatment for this patient. When using aripiprazole to treat BPD, common side effects include insomnia, anxiety and headache (Mobascher, Mobascher, Schlemper, Winterer, \& Malevani, 2006). In BPD, no RCTs for the following atypical antipsychotics have been reported to date: quetiapine, ziprasidone, iloperidone and asenapine.

\section{Discussion/future directions}

The case of patient $M$ provides us with a rich tapestry of clinical history, symptoms and treatments. The field has begun to embrace the necessity for standardization of methods needed to study atypical antipsychotics, mood stabilizers and other agents in the treatment of BPD (Saunders \& Silk, 2009). If followed, the recommendations of Saun- ders and Silk calling for sorting target symptoms into trait dimensions and utilization of identical research templates would be invaluable in unravelling the crazy quilt of current research. Use of much larger sample sizes such as those available in the Collaborative Longitudinal Personality Disorders Study (Sanislow et al., 2002) will also be imperative in realizing the goal of specific researchbased treatment recommendations for BPD.

Many of us have difficult patients similar to $\mathrm{M}$ in our own practices. Currently, we face many challenges in achieving early improvement and lasting relief for these patients. New evidencebased algorithms will hopefully be developed to provide systematic treatment recommendations for use of atypical antipsychotic medications, sorely needed and long overdue, in BPD.

\section{References}

Abraham, P. F., \& Calabrese, J. R. (2008). Evidence-based pharmacologic treatment of borderline personality disorder: A shift from SSRIs to anticonvulsants and atypical antipsychotics? Journal of Affective Disorders, 111, 21-30.

Benedetti, F., Sforzini, L., Colombo, C., Maffei, C., \& Smeraldi, E. (1998). Low dose clozapine in acute and continuation treatment of severe borderline personality disorder. Journal of Clinical Psychiatry, 59, 103-107.

Bogenschutz, M. P., \& Nurnberg, G. (2004). Olanzapine versus placebo in the treatment of borderline personality disorder. Journal of Clinical Psychiatry, 65, 104-109.

Chengappa, K. N., Ebeling, T., Kang, J. S., Levine, J., \& Parepally, H. (1999). Clozapine reduces severe selfmutilation and aggression in psychotic patients with borderline personality disorder. Journal of Clinical Psychiatry, 60, 477-484.

Frankenburg, F. R., \& Zanarini, M. C. (1993). Clozapine treatment of borderline patients: A preliminary study. Comprehensive Psychiatry, 34, 402-405.

Ingenhoven, T., Lafay, P., Rinne, T., Passchier, J., \& Duivenvoorden, H. (2010). Effectiveness of pharmacotherapy for severe personality disorders: Meta-analyses of randomized controlled trials. Journal of Clinical Psychiatry, 71, 14-25.

Koenigsberg, H. W., Reynolds, D., Goodman, M., New, A. S., Mitropoulou, V., Trestman, R. L., Silverman, J., \& Siever, 
L. J. (2003). Risperidone in the treatment of schizotypal personality disorder. Journal of Clinical Psychiatry, 64, 628-634.

Lambertenghi Deliliers, G. (2000). Blood dyscrasias in clozapine-treated patients in Italy. Haematologica, 85, 233-237.

Mobascher, A., Mobascher, J., Schlemper, V., Winterer G., \& Malevani, J. (2006). Aripiprazole pharmacotherapy of borderline personality disorder. Pharmacopsychiatry, 39, 111-112.

Murashita, M., Inoue, T., Kusumi, I., Nakagawa, S., Itoh, K., Tanaka, T., Izumi, T., Hosoda, H., Kangawa, K., \& Koyama, T. (2007). Glucose and lipid metabolism of long-term risperidone monotherapy in patients with schizophenia. Psychiatry and Clinical Neuroscience, 61, 54-58.

Nickel, M. K., Loew, T. H., \& Gil, F. P. (2007). Aripiprazole in the treatment of borderline patients: II, an 18-month follow-up. Psychopharmacology, 191, 1023-1026.

Sanislow, C. A., Grilo, C. M., Morey, L. C., Bender, D. S., Skodol, A. E., Gunderson, J. G., Shea, M. T., Stout, R. L., Zanarini, M. C., \& McGlashan, T. H. (2002). Confirmatory factor analysis of DSM-IV criteria for borderline personality disorder: Findings from the Collaborative Longitudinal Personality Disorders Study. American Journal of Psychiatry, 159, 284-290.
Saunders, E. F. H., \& Silk, K. R. (2009). Personality trait dimensions and the pharmacological treatment of borderline personality disorder. Journal of Clinical Psychopharmacology, 29, 461-467.

Soler, J., Pascual, J. C., Campins, J., Barrachina, J., Puigdemont, D., Alvarez, E., \& Pérez, V. (2005). Doubleblind, placebo-controlled study of dialectical behavior therapy plus olanzapine for borderline personality disorder. American Journal of Psychiatry, 162, 1221-1224.

Soloff, P. (1998). Symptom-oriented psychopharmacology for personality disorders. Journal of Practical Psychiatry and Behavioral Health, 4, 3-11.

Weiden, P. J. (2007). EPS profiles: The atypical antipsychotics are not all the same. Journal of Psychiatric Practice, 13, $13-24$.

Zanarini, M. C., \& Frankenburg, F. R. (2001). Olanzapine treatment of female borderline personality disorder patients: A double-blind, placebo-controlled pilot study. Journal of Clinical Psychiatry, 62, 849-854.

Address correspondence to: Tamara L. Gay, MD, Assistant Professor, Department of Psychiatry, Box 5769, University of Michigan, Rachel Upjohn Building, 4250 Plymouth Road, Ann Arbor, MI 48109-2700, USA. Email: tlgay@med.umich.edu 